\title{
Local reachability of stochastic quantum dynamics with application to feedback control of single-spin systems
}

\author{
Naoki Yamamoto, Hiroshi Machida, Koji Tsumura and Shinji Hara
}

\begin{abstract}
A quantum state subject to a continuous measurement obeys a nonlinear stochastic differential equation called the stochastic master equation. In this paper, we utilize the technique of local reachability developed in control theory to characterize a class of the measurement and the controller in such a way that the local transition of the controlled quantum state is restricted to a low-dimensional manifold. We examine the proposed controller for single-spin systems with two typical measurements.
\end{abstract}

\section{INTRODUCTION}

Since the feedback control method was developed by introducing the continuous measurement [1], the methodologies to control quantum systems have been rapidly progressed. Under the continuous measurement, a finite-dimensional quantum state, which must be a positive semidefinite matrix $P \geq 0$ satisfying $\operatorname{Tr} P=1$, obeys the stochastic master equation (SME) [2], [3], [4]:

$$
\begin{aligned}
& d P=-\mathrm{i}[u H, P] d t+\left[C P C^{*}-\frac{1}{2} C^{*} C P-\frac{1}{2} P C^{*} C\right] d t \\
& +\left[C P+P C^{*}-\left\langle C+C^{*}\right\rangle P\right]\left[d y-\left\langle C+C^{*}\right\rangle d t\right],
\end{aligned}
$$

where $\langle X\rangle:=\operatorname{Tr}(P X)$, and the matrix $C$ is the mathematical expression of a measured physical observable. The notation $*$ means Hermite conjugate. Our objective is to control $P(t)$ by adding an appropriate control Hamiltonian $H$, which is also an Hermite matrix, with a control input $u(t) \in \mathbf{R}$. A control strategy based on the filtering theory [3], [5] is as follows; The measurement data $y(t) \in \mathbf{R}$ is associated with the standard Wiener process [6];

$d y-\left\langle C+C^{*}\right\rangle d t=d W, \quad \mathbf{E}[d W(t)]=0, \mathbf{E}\left[d W(t)^{2}\right]=d t$,

where $\mathbf{E}$ denotes the expectation. Hence, if we know the initial value $P(0)$, updating the filtering equation (1) with the data $y(t)$, we can obtain a perfect knowledge about the time-dependent series of $P(t)$, which eventually enables us to control the quantum system via state-feedback. That is, the objective is to control the stochastic dynamics of the form

$$
\begin{gathered}
d P=-\mathrm{i}[u H, P] d t+\left[C P C^{*}-\frac{1}{2} C^{*} C P-\frac{1}{2} P C^{*} C\right] d t \\
+\left[C P+P C^{*}-\left\langle C+C^{*}\right\rangle P\right] d W
\end{gathered}
$$

N. Yamamoto is with Control and Dynamical Systems, California Institute of Technology, Pasadena, California 91125, USA naoki@cds.caltech. edu

H. Machida, K. Tsumura, and S. Hara are with the Department of Information Physics and Computing, Graduate School of Information Science and Technology, University of Tokyo, Hongo 7-3-1, Bunkyo-ku, Tokyo 113-0033, Japan \{Hiroshi_Machida, Koji_Tsumura, Shinji_Hara\}@ipc.i.u-tokyo.ac.jp with the state-dependent feedback law $u(t)=u(P(t))$. The feedback control scheme mentioned above has been applied to many quantum systems for the following purposes, for example, position control of a particle [2] and spin control of a multi-particle [4], [7], [8]. Although these are indeed great successes, the proposed controllers are ad hoc due to the highly-complicated structure of the SME, and there is still no systematic way to design efficient control Hamiltonian $H$ with input $u(t)$.

On the other hand, control theory has developed a lot of useful tools to deal with a number of complex nonlinear dynamics. One of the key techniques to investigate such complicated systems systematically is reachability analysis [9], which reveals the local transition of state variable driven by system's intrinsic evolution and the controller.

Hence in this paper, we utilize the technique of local reachability to investigate the structure of the SME for general finite dimensional quantum systems. Specifically, we characterize a class of the measurement $C$ and the control Hamiltonian $H$ such that the local transition of the controlled quantum state is restricted to a low-dimensional manifold. In this case, we may design a global controller efficiently by using a visible topology of the dynamics. Moreover, we apply the proposed Hamiltonian to the single-spin system with two typical measurements, the Hermite type and the Nilpotent type. Consequently, in terms of geometrical observations in three dimensional space, the proposed controller will be shown to be efficient for the Nilpotent type measurement.

Notation: $\langle x, y\rangle$ : inner product of vectors $x$ and $y$, i.e., $\langle x, y\rangle:=x^{*} y, \mathcal{L}\left(\mathbf{C}^{n}\right)$ : the set of operators acting on $\mathbf{C}^{n}$, $X^{T}$ : transpose of a complex matrix $X, X \otimes Y$ : Kronecker product of two matrices $X$ and $Y,[X, Y]$ : Lie bracket of $X$ and $Y$, i.e., $[X, Y]:=X Y-Y X$, $\operatorname{dist}\left\{f_{1}, \cdots, f_{m}\right\}$ : the distribution spanned by the vector fields $f_{i}$.

\section{THE THEORY OF LOCAL REACHABILITY}

Consider a Stratonovich type stochastic differential equation of the form

$$
d x=f^{\prime}(x) d t+u(t) g(s) d t+h(x) \circ d W,
$$

where $f^{\prime}(x), g(x)$ and $h(x)$ are analytic vector fields on $\mathbf{C}^{n}$. Now we can directly apply the theory of local reachability [9], which is usually considered for deterministic systems, to the dynamics (3) because it allows the usual differential calculus. Let $\mathcal{P}$ be the smallest distribution which contains $\left\{f^{\prime}(x), g(x), h(x)\right\}$ and is invariant under these vector fields. That is, $\left[f^{\prime}(x), \phi(x)\right]_{\mathbf{L}} \in \mathcal{P},[g(x), \phi(x)]_{\mathbf{L}} \in \mathcal{P}$ and $[h(x), \phi(x)]_{\mathbf{L}} \in \mathcal{P}$ hold for all $\phi(x) \in \mathcal{P}$. Hence for instance 
$\left[h(x),\left[f^{\prime}(x), g(x)\right]_{\mathbf{L}}\right]_{\mathbf{L}} \in \mathcal{P}$ has to hold. Here the Lie product is defined as

$$
[\mu(x), \tau(x)]_{\mathrm{L}}:=\frac{\partial \tau(x)}{\partial x} \mu(x)-\frac{\partial \mu(x)}{\partial x} \tau(x),
$$

where $\partial \tau(x) / \partial x$ is the Jacobian matrix. If $\mathcal{P}$ is non-singular at $x(0)$ and $\operatorname{dim} \mathcal{P}=n_{1}<n$, there exists a coordinates transformation $\Phi: x \rightarrow z$ defined on a neighborhood of $x(0)$ such that the transformed equation is given by

$$
\begin{aligned}
& d z_{1}=f_{1}^{\prime}\left(z_{1}, z_{2}\right) d t+u(t) g_{1}\left(z_{1}, z_{2}\right) d t+h_{1}\left(z_{1}, z_{2}\right) \circ d W, \\
& d z_{2}=0,
\end{aligned}
$$

where $z=z_{1} \oplus z_{2} \in \mathbf{C}^{n_{1}} \oplus \mathbf{C}^{n_{2}}, n_{1}+n_{2}=n$ [9]. The above decomposed system shows that the trajectory starting from $z(0)=z_{1}(0) \oplus z_{2}(0)$ is restricted to the set of the variable $z_{1} \oplus z_{2}(0)$, the $n_{1}$-dimensional manifold $M_{R}$. Therefore, the complement of the manifold $M_{R}$ in $\mathbf{C}^{n}$ is formed by the points where the state variable starting from $z(0)$ does not reach locally with probability one.

\section{LOCAL REACHABLE ANALYSIS FOR GENERAL SME}

We shall utilize the method of local reachability to characterize a pair of the measurement observable $C$ and the control Hamiltonian $H$ such that the controlled quantum state shows a low-dimensional transition. Since it seems to be reasonable to follow the calculating rule of the Lie products using standard column vectors, we introduce a vector representation of a quantum state and aim to derive the vector formed SME. Although some vector representations of a quantum state $P$ can be considered, we utilize a vectorizing technique of a matrix that was originally developed in [10] and has been applied to several studies, e.g., [11], [12].

\section{A. The vector representation of a quantum state}

For an arbitrary vector $\varphi=\left[\varphi_{1} \cdots \varphi_{n}\right]^{T} \in \mathbf{C}^{n}$ and an arbitrary matrix $X=\left(x_{i j}\right) \in \mathcal{L}\left(\mathbf{C}^{n}\right)$, we define $\tilde{\varphi}=\left[\varphi_{1}^{*} \cdots \varphi_{n}^{*}\right]^{T}$ and $\tilde{X}=\left(x_{i j}^{*}\right)$. That is, the tilde operation is defined by $\tilde{\phi}=\left(\phi^{*}\right)^{T}$ and $\tilde{X}=\left(X^{*}\right)^{T}$. Let us consider the product space $\mathbf{C}^{n} \otimes \mathbf{C}^{n}$. When we take $\left\{a_{k}\right\}(k=1, \cdots, n)$ as an orthonormal basis in $\mathbf{C}^{n}$, then $\left\{a_{i} \otimes \tilde{a}_{j}\right\}(i, j=1, \cdots, n)$ becomes an orthonormal basis in $\mathbf{C}^{n} \otimes \mathbf{C}^{n}$. Hence, any vector $\varphi \in \mathbf{C}^{n} \otimes \mathbf{C}^{n}$ can be written by $\boldsymbol{\varphi}=\sum_{i, j=1}^{n} \varphi_{i j} a_{i} \otimes \tilde{a}_{j}$ with scalars $\varphi_{i j}$. We here introduce a specific vector in $\mathbf{C}^{n} \otimes \mathbf{C}^{n}$ given by

$$
\boldsymbol{e}:=\sum_{k=1}^{n} a_{k} \otimes \tilde{a}_{k} .
$$

For example in the case $n=2$, taking $\left\{a_{k}\right\}$ as

$$
a_{1}=(1 / \sqrt{2})[1 \mathrm{i}]^{T}, a_{2}=(1 / \sqrt{2})[1-\mathrm{i}]^{T},
$$

we have $\tilde{a}_{1}=(1 / \sqrt{2})[1-\mathrm{i}]^{T}, \tilde{a}_{2}=(1 / \sqrt{2})[1 \mathrm{i}]^{T}$, and thus

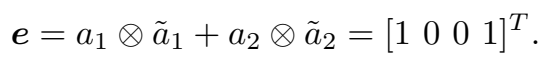

The vector $e$ plays an essential role in order to introduce the vector representation of a quantum state. The following properties are used throughout this paper.
Lemma 1: The vector $e$ is independent of the selection of orthonormal basis, and it satisfies the following properties.

$$
\begin{aligned}
& (X \otimes I) \boldsymbol{e}=\left(I \otimes X^{T}\right) \boldsymbol{e}, \quad \forall X \in \mathcal{L}\left(\mathbf{C}^{n}\right) \\
& \boldsymbol{e}^{*}(X \otimes I) \boldsymbol{e}=\operatorname{Tr} X, \quad \forall X \in \mathcal{L}\left(\mathbf{C}^{n}\right) .
\end{aligned}
$$

Proof: Expanding a matrix $X \in \mathcal{L}\left(\mathbf{C}^{n}\right)$ with respect to an orthonormal basis $\left\{a_{k}\right\}$ as $X=\sum_{i, j} x_{i j} a_{i} a_{j}^{*}$, the left hand side of (6) becomes

$$
\begin{aligned}
& (X \otimes I) \boldsymbol{e}=\left[\sum_{i, j} x_{i j} a_{i} a_{j}^{*} \otimes I\right] \sum_{k} a_{k} \otimes \tilde{a}_{k} \\
& =\sum_{i, j, k} x_{i j}\left\langle a_{j}, a_{k}\right\rangle a_{i} \otimes \tilde{a}_{k}=\sum_{i, j} x_{i j} a_{i} \otimes \tilde{a}_{j} .
\end{aligned}
$$

By the similar way, we can see that the right hand side of Eq. (6) takes the above form. The relation (7) is easy to verify;

$$
e^{*}(X \otimes I) e=\sum_{i, j} a_{i}^{*} \otimes a_{i}^{T}(X \otimes I) a_{j} \otimes \tilde{a}_{j}=\sum_{i} a_{i}^{*} X a_{i},
$$

which is just $\operatorname{Tr} X$. The first assertion in the statement of the lemma can be proved by using the formula (6).

We here define a vector associated with a quantum state $P \in \mathcal{L}\left(\mathbf{C}^{n}\right)$, as

$$
\boldsymbol{p}:=(P \otimes I) \boldsymbol{e} \in \mathbf{C}^{n} \otimes \mathbf{C}^{n} .
$$

Obviously, the vector $\boldsymbol{p}$ is in one-to-one correspondence with $P$, which allows us to call $\boldsymbol{p}$ a quantum state. The equality constraint of a quantum state, $\operatorname{Tr} P=1$, is rewritten as

$$
\langle\boldsymbol{e}, \boldsymbol{p}\rangle=\boldsymbol{e}^{*}(P \otimes I) \boldsymbol{e}=\operatorname{Tr} P=1,
$$

where we have used (7). In the case of $n=2$, the matrix representation of a quantum state $P=\left(p_{i j}\right)$ is given by using (5) as

$$
\boldsymbol{p}=\left[\begin{array}{ll}
p_{11} & p_{12} \\
p_{12}^{*} & p_{22}
\end{array}\right] \otimes\left[\begin{array}{ll}
1 & 0 \\
0 & 1
\end{array}\right] \boldsymbol{e}=\left[\begin{array}{llll}
p_{11} & p_{12} & p_{12}^{*} & p_{22}
\end{array}\right]^{T} .
$$

\section{$B$. The vector representation of the SME}

In this subsection we shall write the SME (2) as a vectorvalued dynamics of $\boldsymbol{p}(t)$. Firstly it is readily seen that the quantity $\langle X\rangle:=\operatorname{Tr}(X P)$ is rewritten by using $\boldsymbol{p}$ as

$$
\langle X\rangle=e^{*}(X \otimes I) p,
$$

since from (7) the right hand side of (10) turns out to be $e^{*}(X P \otimes I) e=\operatorname{Tr}(X P)$. We here introduce an operator from $\mathcal{L}\left(\mathbf{C}^{n}\right)$ to $\mathcal{L}\left(\mathbf{C}^{n} \otimes \mathbf{C}^{n}\right)$ as follows;

$$
\Gamma[X]:=X \otimes I+I \otimes \tilde{X}-\left\langle X+X^{*}\right\rangle I \otimes I .
$$

We sometimes omit the identity matrix in the last term, i.e., $\boldsymbol{\Gamma}[X]=X \otimes I+I \otimes \tilde{X}-\left\langle X+X^{*}\right\rangle$. This operator has the following properties.

Lemma 2: The operator $\boldsymbol{\Gamma}$ satisfies the following properties for any matrices $X, Y \in \mathcal{L}\left(\mathbf{C}^{n}\right)$ and for any scalars $\alpha_{1}, \alpha_{2} \in \mathbf{R}$ and $\beta \in \mathbf{C}$;

$$
\begin{aligned}
& \boldsymbol{\Gamma}\left[\alpha_{1} X+\alpha_{2} Y\right]=\alpha_{1} \boldsymbol{\Gamma}[X]+\alpha_{2} \boldsymbol{\Gamma}[Y], \\
& \boldsymbol{\Gamma}[\beta I]=0, \\
& \boldsymbol{e}^{*} \boldsymbol{\Gamma}[X] \boldsymbol{p}=0, \\
& {[\boldsymbol{\Gamma}[X] \boldsymbol{p}, \boldsymbol{\Gamma}[Y] \boldsymbol{p}]_{\mathrm{L}}=-\boldsymbol{\Gamma}[[X, Y]] \boldsymbol{p} .}
\end{aligned}
$$


Proof: The first and the second properties are obvious. The third is directly verified as follows.

$$
\begin{aligned}
& \boldsymbol{e}^{*} \boldsymbol{\Gamma}[X] \boldsymbol{p}=\boldsymbol{e}^{*}\left[X \otimes I+I \otimes \tilde{X}-\left\langle X+X^{*}\right\rangle\right] \boldsymbol{p} \\
& =\boldsymbol{e}^{*}\left[X \otimes I+\tilde{X}^{T} \otimes I\right] \boldsymbol{p}-\left\langle X+X^{*}\right\rangle\langle\boldsymbol{e}, \boldsymbol{p}\rangle \\
& =\boldsymbol{e}^{*}\left[\left(X+X^{*}\right) \otimes I\right] \boldsymbol{p}-\left\langle X+X^{*}\right\rangle=0,
\end{aligned}
$$

where we have used (6), (9) and (10). In order to prove (15), we give an explicit form of the Jacobian matrix of $\boldsymbol{\Gamma}[X] \boldsymbol{p}$, which is well defined since this is an analytic vector field:

$$
\begin{aligned}
& \frac{\partial \boldsymbol{\Gamma}[X] \boldsymbol{p}}{\partial \boldsymbol{p}}=\frac{\partial}{\partial \boldsymbol{p}}\left[X \otimes I+I \otimes \tilde{X}-\boldsymbol{e}^{*}\left[\left(X+X^{*}\right) \otimes I\right] \boldsymbol{p}\right] \boldsymbol{p} \\
& \quad=X \otimes I+I \otimes \tilde{X}-\left\langle X+X^{*}\right\rangle-\boldsymbol{p} \boldsymbol{e}^{*}\left[\left(X+X^{*}\right) \otimes I\right] \\
& \quad=\boldsymbol{\Gamma}[X]-\boldsymbol{p} \boldsymbol{e}^{*}\left[\left(X+X^{*}\right) \otimes I\right] .
\end{aligned}
$$

Due to the above formula, the left hand side of (15) becomes

$$
\begin{gathered}
\left\{\boldsymbol{\Gamma}[Y]-\boldsymbol{p} \boldsymbol{e}^{*}\left[\left(Y+Y^{*}\right) \otimes I\right]\right\} \boldsymbol{\Gamma}[X] \boldsymbol{p} \\
-\left\{\boldsymbol{\Gamma}[X]-\boldsymbol{p} \boldsymbol{e}^{*}\left[\left(X+X^{*}\right) \otimes I\right]\right\} \boldsymbol{\Gamma}[Y] \boldsymbol{p} \\
=[(Y \otimes I+I \otimes \tilde{Y}),(X \otimes I+I \otimes \tilde{X})] \boldsymbol{p} \\
\quad-\left[\boldsymbol{e}^{*}([Y, X] \otimes I+I \otimes[\tilde{Y}, \tilde{X}]) \boldsymbol{p}\right] \boldsymbol{p} \\
=\boldsymbol{\Gamma}[[Y, X]] \boldsymbol{p}=-\boldsymbol{\Gamma}[[X, Y]] \boldsymbol{p} .
\end{gathered}
$$

This completes the proof.

With the use of the operator $\Gamma$, we get the objective vector representation of the $\mathrm{SME}$;

Lemma 3: The SME (2) is equivalently rewritten by using $\boldsymbol{p} \in \mathbf{C}^{N}$ as

$$
\begin{gathered}
d \boldsymbol{p}=\boldsymbol{f}(\boldsymbol{p}) d t+u(t) \boldsymbol{g}(\boldsymbol{p}) d t+\boldsymbol{h}(\boldsymbol{p}) d W \\
\boldsymbol{f}(\boldsymbol{p}):=\left[C \otimes \tilde{C}-\frac{1}{2} C^{*} C \otimes I-\frac{1}{2} I \otimes \tilde{C}^{*} \tilde{C}\right] \boldsymbol{p} \\
\boldsymbol{g}(\boldsymbol{p}):=\boldsymbol{\Gamma}[-\mathrm{i} H] \boldsymbol{p}=[-\mathrm{i} H \otimes I+\mathrm{i} I \otimes \tilde{H}] \boldsymbol{p} \\
\boldsymbol{h}(\boldsymbol{p}):=\boldsymbol{\Gamma}[C] \boldsymbol{p}=\left[C \otimes I+I \otimes \tilde{C}-\left\langle C+C^{*}\right\rangle\right] \boldsymbol{p} .
\end{gathered}
$$

Proof: Expanding the space $\mathbf{C}^{n}$ to $\mathbf{C}^{n} \otimes \mathbf{C}^{n}$ and multiplying (2) by $e$ from the right, we have

$$
\begin{aligned}
& d(P \otimes I) \boldsymbol{e}=-\mathrm{i} u(t)[(H P-P H) \otimes I] \boldsymbol{e} d t \\
& \quad+\left[\left(C P C^{*}-\frac{1}{2} C^{*} C P-\frac{1}{2} P C^{*} C\right) \otimes I\right] \boldsymbol{e} d t \\
& \quad+\left[\left(C P+P C^{*}-\left\langle C+C^{*}\right\rangle P\right) \otimes I\right] \boldsymbol{e} d W .
\end{aligned}
$$

By virtue of (6), for example we have

$$
\begin{aligned}
& {\left[\left(C P C^{*}\right) \otimes I\right] \boldsymbol{e}=(C P \otimes I)\left(C^{*} \otimes I\right) \boldsymbol{e}} \\
& \quad=(C P \otimes I)\left(I \otimes\left(C^{*}\right)^{T}\right) \boldsymbol{e}=(C P \otimes I)(I \otimes \tilde{C}) \boldsymbol{e} \\
& \quad=(C \otimes \tilde{C})(P \otimes I) \boldsymbol{e}=(C \otimes \tilde{C}) \boldsymbol{p},
\end{aligned}
$$

where we used the definition (8). Similar calculations yield the objective expression of the SME.

The vector-formed dynamics of $\boldsymbol{p}(t)$ satisfies the condition (9). Actually, combining $e^{*} \boldsymbol{f}=0$ with the relations $\boldsymbol{e}^{*} \boldsymbol{g}=$ $\boldsymbol{e}^{*} \boldsymbol{\Gamma}[-\mathrm{i} H] \boldsymbol{p}=0$ and $\boldsymbol{e}^{*} \boldsymbol{h}=\boldsymbol{e}^{*} \boldsymbol{\Gamma}[C] \boldsymbol{p}=0$, which are from
(14), we have $d\langle\boldsymbol{e}, \boldsymbol{p}\rangle=0$. That is, $\langle\boldsymbol{e}, \boldsymbol{p}(t)\rangle$ take a constant value. The next subject is to obtain the explicit form of the corresponding Stratonovich SDE:

$$
d \boldsymbol{p}=\left[\boldsymbol{f}-\frac{1}{2} \frac{\partial \boldsymbol{h}}{\partial \boldsymbol{p}} \boldsymbol{h}\right](\boldsymbol{p}) d t+u(t) \boldsymbol{g}(\boldsymbol{p}) d t+\boldsymbol{h}(\boldsymbol{p}) \circ d W .
$$

Note again that the Jacobian matrix $\partial \boldsymbol{h}(\boldsymbol{p}) / \partial \boldsymbol{p}$ can be defined since $\boldsymbol{h}(\boldsymbol{p})$ is an analytic vector field.

Lemma 4: The Stratonovich SME is given by

$$
\begin{gathered}
d \boldsymbol{p}=\boldsymbol{f}^{\prime}(\boldsymbol{p}) d t+u(t) \boldsymbol{g}(\boldsymbol{p}) d t+\boldsymbol{h}(\boldsymbol{p}) \circ d W, \\
\boldsymbol{f}^{\prime}(\boldsymbol{p}):=-\boldsymbol{\Gamma}\left[C^{\prime}\right] \boldsymbol{p}+\left\langle C+C^{*}\right\rangle \boldsymbol{\Gamma}[C] \boldsymbol{p}, \\
C^{\prime}:=\left(C^{*} C+C^{2}\right) / 2,
\end{gathered}
$$

where the vector fields $\boldsymbol{g}(\boldsymbol{p})$ and $\boldsymbol{h}(\boldsymbol{p})$ are given in Lemma 3.

Proof: From (16), we have

$$
\begin{gathered}
\frac{\partial \boldsymbol{h}(\boldsymbol{p})}{\partial \boldsymbol{p}} \boldsymbol{h}(\boldsymbol{p})=\left\{\boldsymbol{\Gamma}[C]-\boldsymbol{p} \boldsymbol{e}^{*}\left[\left(C+C^{*}\right) \otimes I\right]\right\} \boldsymbol{\Gamma}[C] \boldsymbol{p} \\
=\boldsymbol{\Gamma}[C]^{2} \boldsymbol{p}-\left\{\boldsymbol{e}^{*}\left[\left(C+C^{*}\right) \otimes I\right] \boldsymbol{\Gamma}[C] \boldsymbol{p}\right\} \boldsymbol{p} .
\end{gathered}
$$

The first and the second term are respectively calculated into

$$
\begin{gathered}
\boldsymbol{\Gamma}[C]^{2}=(C \otimes I+I \otimes \tilde{C})^{2}+\left\langle C+C^{*}\right\rangle^{2} \\
\quad-2\left\langle C+C^{*}\right\rangle(C \otimes I+I \otimes \tilde{C}) \\
=C^{2} \otimes I+2 C \otimes \tilde{C}+I \otimes \tilde{C}^{2}-\left\langle C+C^{*}\right\rangle^{2} \\
\quad-2\left\langle C+C^{*}\right\rangle \boldsymbol{\Gamma}[C], \\
\boldsymbol{e}^{*}\left[\left(C+C^{*}\right) \otimes I\right] \boldsymbol{\Gamma}[C] \boldsymbol{p} \\
=\boldsymbol{e}^{*}[C \otimes I+I \otimes \tilde{C}]\left[C \otimes I+I \otimes \tilde{C}-\left\langle C+C^{*}\right\rangle\right] \boldsymbol{p} \\
=\left\langle C^{2}+2 C^{*} C+C^{* 2}\right\rangle-\left\langle C+C^{*}\right\rangle^{2} .
\end{gathered}
$$

Consequently, we have

$$
\begin{aligned}
& \frac{\partial \boldsymbol{h}(\boldsymbol{p})}{\partial \boldsymbol{p}} \boldsymbol{h}(\boldsymbol{p})=\left[C^{2} \otimes I+2 C \otimes \tilde{C}+I \otimes \tilde{C}^{2}\right] \boldsymbol{p} \\
& \quad-\left\langle C+C^{*}\right\rangle^{2} \boldsymbol{p}-2\left\langle C+C^{*}\right\rangle \boldsymbol{\Gamma}[C] \boldsymbol{p}+\left\langle C+C^{*}\right\rangle^{2} \boldsymbol{p} \\
& \quad-\left\langle C^{2}+2 C^{*} C+C^{* 2}\right\rangle \boldsymbol{p} \\
& =\boldsymbol{\Gamma}\left[C^{2}\right] \boldsymbol{p}-2\left\langle C+C^{*}\right\rangle \boldsymbol{\Gamma}[C] \boldsymbol{p}+2\left[C \otimes \tilde{C}-\left\langle C^{*} C\right\rangle\right] \boldsymbol{p},
\end{aligned}
$$

which leads to

$$
\begin{gathered}
\boldsymbol{f}(\boldsymbol{p})-\frac{1}{2} \frac{\partial \boldsymbol{h}(\boldsymbol{p})}{\partial \boldsymbol{p}} \boldsymbol{h}(\boldsymbol{p})=-\frac{1}{2} \boldsymbol{\Gamma}\left[C^{2}\right] \boldsymbol{p}+\left\langle C+C^{*}\right\rangle \boldsymbol{\Gamma}[C] \boldsymbol{p} \\
-\frac{1}{2}\left[C^{*} C \otimes I+I \otimes \tilde{C}^{*} \tilde{C}-\left\langle C^{*} C+\left(C^{*} C\right)^{*}\right\rangle\right] \boldsymbol{p} \\
=-\frac{1}{2} \boldsymbol{\Gamma}\left[C^{*} C\right] \boldsymbol{p}-\frac{1}{2} \boldsymbol{\Gamma}\left[C^{2}\right] \boldsymbol{p}+\left\langle C+C^{*}\right\rangle \boldsymbol{\Gamma}[C] \boldsymbol{p} .
\end{gathered}
$$

Hence, the property (12) concludes the proof.

\section{The local reachability of the SME}

Let us firstly compute the Lie product of $h$ and $\boldsymbol{f}^{\prime}$. Noting that for any analytic function $a(\boldsymbol{p})$ and analytic vector fields $\boldsymbol{f}_{1}(\boldsymbol{p})$ and $\boldsymbol{f}_{2}(\boldsymbol{p})$,

$$
\left[a \boldsymbol{f}_{1}, \boldsymbol{f}_{2}\right]_{\mathrm{L}}=a\left[\boldsymbol{f}_{1}, \boldsymbol{f}_{2}\right]_{\mathrm{L}}-\left\langle\frac{\partial a}{\partial \boldsymbol{p}}, \boldsymbol{f}_{2}\right\rangle \boldsymbol{f}_{1}
$$


holds, we have

$$
\begin{aligned}
& {\left[\boldsymbol{f}^{\prime}, \boldsymbol{h}\right]_{\mathrm{L}}=-\left[\boldsymbol{\Gamma}\left[C^{\prime}\right] \boldsymbol{p}, \boldsymbol{\Gamma}[C] \boldsymbol{p}\right]_{\mathrm{L}}+\left[\left\langle C+C^{*}\right\rangle \boldsymbol{h}, \boldsymbol{h}\right]_{\mathrm{L}}} \\
& =\frac{1}{2} \boldsymbol{\Gamma}\left[\left[C^{*} C, C\right]\right] \boldsymbol{p}-\left[\left\langle C^{2}+2 C^{*} C+C^{* 2}\right\rangle-\left\langle C+C^{*}\right\rangle^{2}\right] \boldsymbol{h},
\end{aligned}
$$

where we have used (15) and (17). We here consider the system such that $\left[\boldsymbol{f}^{\prime}, \boldsymbol{h}\right]_{\mathrm{L}}$ is included into the distribution spanned by $\boldsymbol{f}^{\prime}$ and $\boldsymbol{h}$. In this case, from the theory of local reachability there exist a local mapping that transforms the dynamics to a decomposed one where the $n^{2}-2$ variables take constants. (Note that $\boldsymbol{p}$ is an $n^{2}$-dimensional vector.) That is, we can describe the dynamics by only two parameters. A simple sufficient condition for such kind of systems to exist is given as follows.

Theorem 1: $\quad$ Suppose there exist $a_{0} \in \mathbf{C}$ and $a_{1}, a_{2} \in \mathbf{R}$ such that

$$
\left[C^{*} C, C\right]=a_{0} I+a_{1} C+a_{2} C^{\prime}
$$

is satisfied. Then, $\left[\boldsymbol{f}^{\prime}, \boldsymbol{h}\right]_{\mathrm{L}}(\boldsymbol{p}) \in \operatorname{dist}\left\{\boldsymbol{f}^{\prime}, \boldsymbol{h}\right\}$ holds.

Proof: From (12) and (13) we have

$$
\begin{aligned}
{\left[\boldsymbol{f}^{\prime}, \boldsymbol{h}\right]_{\mathrm{L}}(\boldsymbol{p})=} & \frac{1}{2} \boldsymbol{\Gamma}\left[a_{0} I+a_{1} C+a_{2} C^{\prime}\right] \boldsymbol{p} \\
& \left.-\left[\left\langle C^{2}+2 C^{*} C+C^{* 2}\right\rangle-\left\langle C+C^{*}\right\rangle^{2}\right)\right] \boldsymbol{h} \\
= & \frac{a_{1}}{2} \boldsymbol{h}+\frac{a_{2}}{2}\left[\left\langle C+C^{*}\right\rangle \boldsymbol{h}-\boldsymbol{f}^{\prime}\right] \\
& \left.-\left[\left\langle C^{2}+2 C^{*} C+C^{* 2}\right\rangle-\left\langle C+C^{*}\right\rangle^{2}\right)\right] \boldsymbol{h},
\end{aligned}
$$

which is obviously included in $\operatorname{dist}\left\{\boldsymbol{f}^{\prime}, \boldsymbol{h}\right\}$.

A remarkable fact is that many typical continuous measurements have the above striking feature. For example, when $C$ is included in the following sets:

\section{(i) Hermite (ii) Skew Hermite (iii) Specific Nilpotent,}

the condition in Theorem 1 is satisfied. Actually, if $C$ is in (i) or (ii), it satisfies $\left[C^{*} C, C\right]=0$. We can find many kinds of Hermite type measurements [2], [4], [7], [13], [14], and some skew-Hermite type measurement [5]. The Nilpotent type measurements usually appear in the case of a damped atom [15], [16] or modified damped atoms [17]. In these studies, the measurements are respectively given as follows;

$$
C_{1}=\left[\begin{array}{ll}
0 & 0 \\
1 & 0
\end{array}\right], \quad C_{2}=\left[\begin{array}{cccc}
0 & \mathrm{i} & \mathrm{i} & 0 \\
0 & 0 & 0 & 0 \\
0 & 0 & 0 & 0 \\
0 & -\mathrm{i} & -\mathrm{i} & 0
\end{array}\right]
$$

Actually, they satisfy $\left[C_{1}^{*} C_{1}, C_{1}\right]=-C_{1}$ and $\left[C_{2}^{*} C_{2}, C_{2}\right]=$ $-4 C_{2}$.

Let us consider a system such that $C$ satisfies the condition in Theorem 1. Then, we can expect that an efficient global control of the SME is possible by using a controller that realizes a low-dimensional transition of the state. Actually, for the control problem of a two-spin system [17], a twodimensional description of the dynamics has played a central role in designing stabilizing controllers.

Therefore, we here aim to obtain a condition of the control Hamiltonian in such a way that the above-mentioned situation is attained. A typical control Hamiltonian is obtained from the condition where both $[\boldsymbol{h}, \boldsymbol{g}]_{\mathrm{L}}(\boldsymbol{p})$ and $\left[\boldsymbol{f}^{\prime}, \boldsymbol{g}\right]_{\mathrm{L}}(\boldsymbol{p})$ are included in the distribution $\operatorname{dist}\left\{\boldsymbol{f}^{\prime}, \boldsymbol{g}, \boldsymbol{h}\right\}$. Let us calculate the Lie products. It is easy to get

$$
[\boldsymbol{h}, \boldsymbol{g}]_{\mathrm{L}}=[\boldsymbol{\Gamma}[C] \boldsymbol{p}, \boldsymbol{\Gamma}[-\mathrm{i} H] \boldsymbol{p}]_{\mathrm{L}}=-\boldsymbol{\Gamma}[[-\mathrm{i} H, C]] \boldsymbol{p} .
$$

A similar calculation yields

$$
\begin{aligned}
{\left[\boldsymbol{f}^{\prime}, \boldsymbol{g}\right]_{\mathrm{L}}=- } & {\left[\boldsymbol{\Gamma}\left[C^{\prime}\right] \boldsymbol{p}, \boldsymbol{\Gamma}[-\mathrm{i} H] \boldsymbol{p}\right]_{\mathrm{L}}+\left[\left\langle C+C^{*}\right\rangle \boldsymbol{h}, \boldsymbol{g}\right]_{\mathrm{L}} } \\
=- & \boldsymbol{\Gamma}\left[\left[-\mathrm{i} H, C^{\prime}\right]\right] \boldsymbol{p}+\left\langle C+C^{*}\right\rangle \boldsymbol{\Gamma}[[-\mathrm{i} H, C]] \boldsymbol{p} \\
& +\left\langle[-\mathrm{i} H, C]+[-\mathrm{i} H, C]^{*}\right\rangle \boldsymbol{\Gamma}[C] \boldsymbol{p} .
\end{aligned}
$$

From the same reason as that in Theorem 1, we have the objective condition.

Theorem 2: $\quad$ Suppose there exist $a_{0} \in \mathbf{C}$ and $a_{1}, a_{2}, a_{3} \in$ $\mathbf{R}$ such that

$$
\begin{aligned}
& {[-\mathrm{i} H, C]=a_{0} I+a_{1} C+a_{2} C^{\prime}+a_{3}(-\mathrm{i} H),} \\
& {\left[-\mathrm{i} H, C^{\prime}\right]=a_{0} I+a_{1} C+a_{2} C^{\prime}+a_{3}(-\mathrm{i} H)}
\end{aligned}
$$

are satisfied. Then, $[\boldsymbol{h}, \boldsymbol{g}]_{\mathrm{L}}(\boldsymbol{p}) \in \operatorname{dist}\left\{\boldsymbol{f}^{\prime}, \boldsymbol{g}, \boldsymbol{h}\right\}$ and $\left[\boldsymbol{f}^{\prime}, \boldsymbol{g}\right]_{\mathrm{L}}(\boldsymbol{p}) \in \operatorname{dist}\left\{\boldsymbol{f}^{\prime}, \boldsymbol{g}, \boldsymbol{h}\right\}$ hold.

\section{ApPliCATION TO THE FEEDBACK CONTROL OF SINGLE-SPIN SYSTEMS}

In this section we apply the results obtained so far to the single-spin, the simple yet important quantum system with dimension $n=2$. Specifically, we aim to control the system by adding a control Hamiltonian which satisfies the condition in Theorem 2. We consider two typical measurements:

$$
C_{\mathrm{H}}=\left[\begin{array}{cc}
1 & 0 \\
0 & -1
\end{array}\right], \quad C_{\mathrm{N}}=\left[\begin{array}{ll}
0 & 0 \\
1 & 0
\end{array}\right],
$$

where here the measurement strength is set to unity for simplicity. The former corresponds to the standard measurement of the spin along an axis (z-axis). The latter, which has already appeared in (19), represents a measurement accompanied with damping. We now introduce a convenient parameterization of the quantum state $P \in \mathcal{L}\left(\mathbf{C}^{2}\right)$ as follows:

$$
P=\frac{1}{2}\left[\begin{array}{cc}
1+z & x-\mathrm{i} y \\
x+\mathrm{i} y & 1-z
\end{array}\right],
$$

where the real parameters $\boldsymbol{x}=(x, y, z)$ have to satisfy the condition $x^{2}+y^{2}+z^{2} \leq 1$ due to $P \geq 0$. Here we follow a standard control objective [4], [5]: stabilize the dynamics at the point $\boldsymbol{s}_{\text {up }}=(0,0,1)$, which corresponds to so-called the spin-up state, $S_{\text {up }}=\operatorname{diag}\{1,0\}$.

\section{A. Hermite case}

The SME with the matrix $C_{\mathrm{H}}$ is written by using $\boldsymbol{x}$ as

$$
\begin{aligned}
& d x=-(x / 2) d t+u(t)(p y+q z) d t-x z d W, \\
& d y=-(y / 2) d t+u(t)(-p x+r z) d t-y z d W, \\
& d z=-u(t)(q x+r y) d t+\left(1-z^{2}\right) d W .
\end{aligned}
$$

The real parameters $p, q$, and $r$ are defined by

$$
p:=h_{22}-h_{11}, q:=-2 \operatorname{Im}\left(h_{12}\right), r:=-2 \operatorname{Re}\left(h_{12}\right),
$$




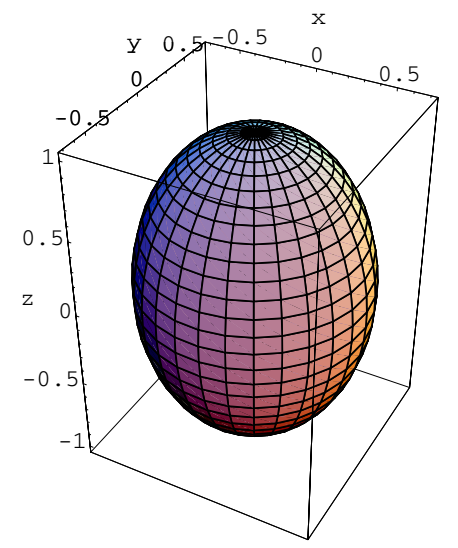

Fig. 1. The manifold in which the quantum state obeying the SME associated with $C_{\mathrm{H}}$ is limited. The parameter is $k_{1}=1 / 2$.

where $\left(h_{i j}\right)$ is the $(i, j)$-element of the control Hamiltonian $H$. As seen in the previous section, the measurement matrix $C_{\mathrm{H}}$ satisfies the condition in Theorem 1, and thus, there exists a two-dimensional manifold to which $\boldsymbol{x}$ obeying the autonomous dynamics is restricted. This manifold is easily obtained from the following computation:

$$
d\left(\frac{1-z^{2}}{x^{2}+y^{2}}\right)=\frac{1-(z+d z)^{2}}{(x+d x)^{2}+(y+d y)^{2}}-\frac{1-z^{2}}{x^{2}+y^{2}}=0 .
$$

This implies that $\left(1-z^{2}\right) /\left(x^{2}+y^{2}\right)$ is invariant without respect to the stochastic noise $W(t)$. That is, $\boldsymbol{x}(t)$ is always limited to the ellipse $x^{2}+y^{2}=k_{1}\left(1-z^{2}\right)$, where $k_{1}$ is constant value determined from the initial state. Figure 1 illustrates an example of the ellipse. We also see that $y / x$ is invariant. As consequence, $\boldsymbol{x}$ is not allowed to evolve in twodimensional space, but is subject to the more strict limitation to a one-dimensional intersection between the plain $y=k_{2} x$ and the ellipse $x^{2}+y^{2}=k_{1}\left(1-z^{2}\right)$.

We now derive the control Hamiltonian which satisfies the condition in Theorem 2. Due to $C^{\prime}=I$, the second condition automatically holds. The first condition is written by

$2 \mathrm{i}\left[\begin{array}{cc}0 & h_{12} \\ -h_{12}^{*} & 0\end{array}\right]=\left(a_{0}+a_{2}\right) I+a_{1} C-\mathrm{i} a_{3}\left[\begin{array}{ll}h_{11} & h_{12} \\ h_{12}^{*} & h_{22}\end{array}\right]$,

which leads to $h_{12}=0$, i.e., $q=r=0$. Then, the control Hamiltonian causes the rotation of the state $\boldsymbol{x}$ around the $z$-axis. This implies that the controlled state is still restricted to the ellipse and shows the two-dimensional transition.

Does this controller achieve our control object, $\boldsymbol{x} \rightarrow \boldsymbol{s}_{\text {up }}$ by using some feedback laws? Unfortunately, we immediately get a negative answer because the control Hamiltonian only rotates $\boldsymbol{x}$ around $z$-axis and cannot control $z$-element itself. Actually, the dynamics of $z(t)$, which is given by $d z=$ $\left(1-z^{2}\right) d W$, shows an undesirable behavior as follows; Let us consider a function $V(z)=1-z^{2} \geq 0$. Then, we easily have $\mathcal{L} V=-V^{2} \leq 0$, which leads to $\lim _{t \rightarrow \infty} \mathcal{L} V=0$ from Lemma 5 in Appendix. Therefore, the uncontrollable convergence $z(t) \rightarrow-1$ can occur as shown in Figure 2 . This point $s_{\text {down }}=(0,0,-1)$ corresponds to the spin-down

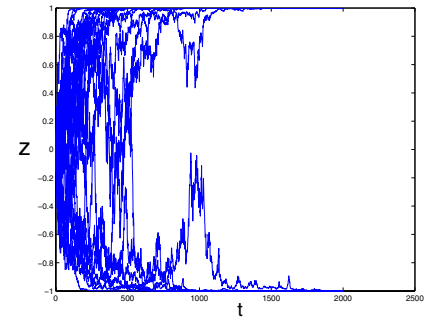

Fig. 2. The stochastic bifurcation of $z(t)$. The initial state is $z(0)=0$.

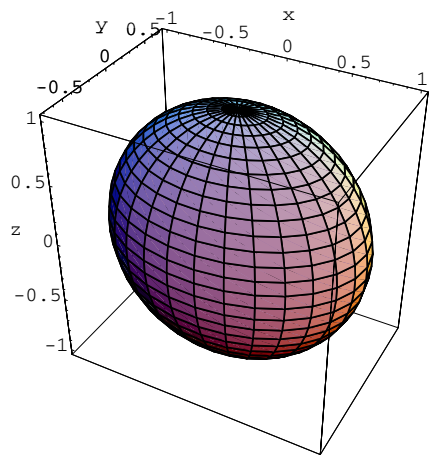

Fig. 3. The manifold in which the quantum state obeying the SME associated with $C_{\mathrm{N}}$ is limited. The parameter is $k_{3}=-2$.

state $S_{\text {down }}=\operatorname{diag}\{0,1\}$. These observations indicate that another control target cannot be also stabilized since $\boldsymbol{x}$ must converge into $s_{\text {up }}$ or $s_{\text {down }}$. As a result, we have to conclude that the proposed controller is not appropriate for the singlespin system with the Hermite type measurement.

\section{B. Nilpotent case}

We next consider the nilpotent type measurement $C_{\mathrm{N}}$. The variable $\boldsymbol{x}$ obeys the following equation;

$$
\begin{aligned}
& d x=-(x / 2) d t+u(t)(p y+q z) d t+\left(-x^{2}+z+1\right) d W, \\
& d y=-(y / 2) d t+u(t)(-p x+r z) d t-x y d W, \\
& d z=-(z+1) d t-u(t)(q x+r y) d t-x(z+1) d W . \text { (22) }
\end{aligned}
$$

Similar to the Hermite type measurement, the autonomous SME is always constrained to evolve on a two-dimensional manifold since $C_{\mathrm{N}}$ satisfies the condition in Theorem 1 . Actually, the variable $k_{3}=\left(x^{2}+z^{2}-1\right) / y^{2}$ satisfies $d\left(k_{3}\right)=0$, which leads to that $\boldsymbol{x}(t)$ is always on the manifold $x^{2}+z^{2}-k_{3} y^{2}=1$. Figure 3 shows this ellipse.

Let us consider the Hamiltonian that satisfies the condition in Theorem 2. Although we here omit the actual derivation for the condition, it is easy to get $p=r=0$, which corresponds to the Hamiltonian rotating the state around the $y$-axis. Obviously, the controlled variable is still limited into the ellipse $x^{2}+z^{2}-k_{3} y^{2}=1$, and the controller does not have ability to manipulate $y$-element. Actually, taking a function $V(y)=y^{2}$, we have $\mathcal{L} V=-y^{2}\left(1-x^{2}\right) \leq 0$ without respect to the control input. From Lemma 5 this implies $\mathcal{L} V \rightarrow 0$, or equivalently $y \rightarrow 0$. (Note that $x^{2} \rightarrow 1$ implies $y \rightarrow 0$.) Then, the ellipse $x^{2}+z^{2}-k_{3} y^{2}=1$ reduces to a circle $x^{2}+z^{2}=1$. Therefore, it turns out that the 
controlled dynamics becomes one-dimensional, which allows us to parametrize the variable by $(x, z)=(\sin \theta, \cos \theta)$. Then, we have a dynamics on $-\pi \leq \theta<\pi$ :

$$
d \theta=\left[u(t)+\frac{1}{2} \sin \theta \cos \theta+\sin \theta\right] d t+(\cos \theta+1) d W .
$$

A remarkable fact is that the dynamics has only one equilibrium point $\theta=\pi$, which corresponds to the spin down state $S=\operatorname{diag}\{0,1\}$, whereas our control target corresponds to a non-equilibrium point $\theta=0$. Although the strict convergence of the state into $\theta=0$ is impossible, we instead aim to let the state go as close to the target as possible. This control objective is attained as follows;

Proposition 3: For the dynamics (23), let us consider a feedback law $u(t)=-M \sin (\theta(t) / 2)$, which is discontinuous at $\theta=-\pi$. Then, the following specification is attained;

$$
\mathbf{E}\left[\sin ^{2} \frac{\theta(t)}{4}\right] \leq \mathrm{e}^{-(M / 2) t} \sin ^{2} \frac{\phi(0)}{4}+\frac{5}{8 M} .
$$

Proof: The function $V(\theta)=\sin ^{2}(\theta / 4)$ satisfies

$$
\begin{aligned}
\mathcal{L} V & =\left[u+\frac{1}{2} \sin \theta \cos \theta+\sin \theta\right] \frac{\partial V}{\partial \theta}+\frac{1}{2}(\cos \theta+1)^{2} \frac{\partial^{2} V}{\partial \theta^{2}} \\
& =-\frac{M}{2}(1+x) V-\frac{1}{4} x\left(x-\frac{1}{2}\right)^{2}+\frac{5}{16} x
\end{aligned}
$$

where $x:=\cos (\theta / 2)$. Noting that $0 \leq x \leq 1$ due to $-\pi \leq \theta<\pi$, the above equation can be evaluated as $\mathcal{L} V \leq(M / 2) V+5 / 16$, which leads to the assertion from Lemma 6.

Clearly, a high-gain feedback control attains a sufficient suppression of the variance-like function. That is, $\mathbf{E}\left[\sin ^{2}(\theta(t) / 4)\right]$ is close to zero as $t \rightarrow \infty$ if $M \gg 1$, which means that $\theta=0$ is approximately achieved.

\section{CONCLUSION}

In this paper, we have considered the highly-complicated quantum system conditioned on the continuous measurement. The key property in order to deal with the complexity is the stochastic local reachability defined by the analogous way to the deterministic case. Through the reachability analysis base on the vector representation of the quantum state, we have characterized a class of the measurement such that the local transition of the state obeying the controlled dynamics is restricted to a three-dimensional manifold. Then, for the single-spin system with a specific type of the measurement, the Nilpotent measurement, we could design a global controller by selecting a control Hamiltonian satisfying the above requirement. This result suggests that the same kind of controller will work well even for a multi-spin system when we use the Nilpotent-type measurement.

\section{APPENDIX}

Consider an Ito SDE on $\mathbf{R}^{n}$ of the form

$$
d x=f(x) d t+g(x) d W,
$$

where $f(x)$ and $g(x)$ are smooth vector fields on $\mathbf{R}^{n}$. Suppose that the dynamics (24) has an equilibrium point $x_{o}$ satisfying $f\left(x_{o}\right)=g\left(x_{o}\right)=0$. Define the infinitesimal generator of $x$ as

$$
\mathcal{L}:=\sum_{i} f_{i} \frac{\partial}{\partial x_{i}}+\frac{1}{2} \sum_{i, j}\left(g g^{T}\right)_{i j} \frac{\partial^{2}}{\partial x_{i} \partial x_{j}} .
$$

Then we have the following fact [18], [19];

Lemma 5: Let $G$ be a bounded invariant set with respect to the solution of (24) and $x_{o} \in G$. Suppose there exists a function $V: G \rightarrow \mathbf{R}_{+}$such that $\mathcal{L} V(x) \leq 0$ holds for all $x \in G$. Then we have $\lim _{t \rightarrow \infty} \mathcal{L} V(x)=0$ a.s..

Lemma 6: Suppose there exist a $C^{2}$ function $V: \mathbf{R}^{n} \rightarrow$ $\mathbf{R}_{+}$, constants $\alpha>0$ and $\beta>0$ such that $\mathcal{L} V(x) \leq$ $-\alpha V(x)+\beta$ for all $x \in \mathbf{R}^{n}$ and $t \geq 0$. Then, there is a unique solution of (24) for each $x(0)$ and it satisfies

$$
\mathbf{E}[V(x(t))] \leq V(x(0)) \mathrm{e}^{-\alpha t}+\beta / \alpha .
$$

\section{REFERENCES}

[1] C. M. Caves and G. J. Milburn: Quantum-mechanical model for continuous position measurements, Phys. Rev. A36, 5543 (1987).

[2] A. C. Doherty and K. Jacobs: Feedback control of quantum systems using continuous state estimation, Phys. Rev. A60, 2700 (1999).

[3] V. P. Belavkin: Quantum stochastic calculus and quantum nonlinear fi ltering, J. Multivariate Anal., vol.42, pp.171-201 (1992).

[4] R. van Handel, J. K. Stockton and H. Mabuchi: Feedback control of quantum state reduction, submitted to IEEE Trans. Automat. Contr..

[5] L. Bouten, S. Edwards and V.P. Belavkin: Bellman equations for optimal feedback control of qubit states, e-print quant-ph/0407192 (2004).

[6] C. W. Gardiner: Handbook of Stochastic Methods, Springer, Berlin (1983).

[7] J. K. Stockton, R. van Handel and H. Mabuchi: Deterministic Dicke state preparation with continuous measurement and control, Phys. Rev. A70, 022106 (2004).

[8] N. Yamamoto, K. Tsumura and S. Hara: Feedback control of quantum entanglement in a two-spin system, to appear, CDC-ECC'05.

[9] A. Isidori, Nonlinear Control Systems, Springer, 1990.

[10] R. A. Horn and C. R. Johnson: Topics in matrix analysis, Cambridge University Press, Cambridge (1991).

[11] N. Yamamoto, S. Hara and K. Tsumura: Suboptimal quantum-errorcorrecting procedure based on semidefi nite programming, Phys. Rev. A71, 022322 (2005).

[12] G. M. D’Ariano and P. Lo Presti, Phys. Rev. A64, 042308 (2001).

[13] J. M. Geremia, J. K. Stockton and H. Mabuchi: Real-time quantum feedback control of atomic spin-squeezing, Science, 304, 270 (2004).

[14] L. K. Thomsen, S. Mancini and H. M. Wiseman: Spin squeezing via quantum feedback, Phys. Rev. A65, 061801 (2001).

[15] J. Wang and H. M. Wiseman, Feedback stabilization of an arbitrary pure state of a two-level atom, Phys. Rev. A, vol.64, p.063810, 2001.

[16] J. Wang, H. M. Wiseman and G. J. Milburn, Dynamical creation of entanglement by homodyne-mediated feedback, e-print quant$\mathrm{ph} / 0409154$ (2004)

[17] N. Yamamoto: Parametrization of the feedback Hamiltonian realizing a pure steady-state, Phys. Rev. A, vol.64, p.024104, 2001.

[18] R. Z. Has'minskii, Stochastic stability of differential equations, Sijthoff \& Noordhoff (1980).

[19] H. Deng, M. Krstic and R. J. Williams, Stabilization of stochastic nonlinear systems driven by noise of unknown covariance, IEEE Trans. Automat. Contr., vol.46, pp.1237-1253, 2001. 